\title{
Geometric and Kinematic Analysis of Deployable Doubly Ruled Hyperboloids
}

\author{
Biçim Değiştirebilen Çift Yönlü Çizel Yüzey Hiperboloidlerin \\ Geometrik ve Kinematik Analizi
}

Feray MADEN, ${ }^{1}$ Koray KORKMAZ ${ }^{2}$

ABSTRACT

This paper aims to develop deployable doubly ruled hyperboloid surfaces that can be used in architectural applications. First, the study systematically analyzes the geometric principles of hyperboloids generated by the ruled surface generation method and examines the morphology to generate different types of hyperboloids. Then, a method is introduced to construct deployable doubly ruled hyperboloid surfaces. The study demonstrates that deployment behavior of the hyperboloid is directly related to joint types used at the intersection points of the bars. Based on kinematic analysis, the study establishes that deployable hyperboloids with a single degree of freedom can be constructed only by revolute and spherical joints. Finally, various hyperboloids having different number of intersections are constructed according to the proposed joint types and their deployment capabilities are discussed.

Keywords: Deployable hyperboloids; doubly-ruled surfaces; kinetic structures; structural mechanisms.

ÖZ

Bu yazının amacı, mimari uygulamalarda kullanılabilecek biçim değiştirebilen çift yönlü çizel hiperboloid yüzeyler geliştirmektir. İlk olarak, çizel yüzey türetme metoduyla üretilen hiperboloidlerin geometrik prensipleri sistematik olarak analiz edilmekte ve farklı tiplerde türetilmesi için morfolojisi incelenmektedir. Daha sonra, biçim değiştirebilen hiperboloid yüzeylerin inşası için geliştirilen bir metot tanıtılmaktadır. Çalışma, hiperboloidlerin hareket davranışlarının çubuk elemanların kesişim noktalarında yer alan bağlantı tipleriyle doğrudan ilişkili olduğunu göstermektedir. Tek serbestlik dereceli hiperboloidlerin yalnızca döner ve küresel mafsal kullanarak inşa edilebildiği kinematik analizle kanıtlanmaktadır. Son olarak, önerilen mafsal tiplerine göre farklı sayıda kesişim sayısına sahip hiperboloidler türetilmekte ve bunların hareket kabiliyetleri tartışılmaktadır.

Anahtar sözcükler: Biçim değiştirebilen hiperboloidler; çift-çizel yüzeyler; kinetik strüktürler; strüktürel mekanizmalar. 


\section{Introduction}

The geometrical simplicity and structural advantages of doubly curved anticlastic surfaces opened a wide range of applications in architecture and engineering. Pioneered by Vladimir Shukhov, Antoni Gaudi and Félix Candela, anticlastic surfaces such as hyperboloid of one sheet and hyperbolic paraboloid (hypar) have been used for cooling towers, oil and water storage tanks, radio and transmission masts, offshore structures and other industrial premises. Over the last century, various different types of structures in the forms of hyperboloid and hypar have been built around the world.

Hyperboloid geometry's surface generation method and its form based structural behavior played a fundamental role in constructing hyperboloid structures. Since the straight-line generators can form the anticlastic surface, concrete shell structures have been easily constructed by using straight wooden boards, which not only simplified the formwork but also provided efficient use of construction materials. Hence, various elegant structures with different curvatures could be realized to span over large areas. Likewise, steel and timber grid shells made with lattice of straight beams have been developed to obtain not only aesthetically pleasing structures but also structurally efficient design solutions. The impressive strength and stiffness of the hyperboloid geometry has enabled to support large loads at the top of the hyperboloid tower structures.

The major advantages of the hyperboloid lattice structures based on their spatial, structural and constructional efficiencies attracted the attention of modern architects. Even though these structures have been mostly used in purpose driven industrial architecture in the nineteenth and twentieth centuries, different functions have been proposed for them in the last decades by covering the structures with glass either inside or outside. Manchester Corporation Street Footbridge can be shown as an example of this usage (Figure 1a). Designed by Hodder \& Partners and built in 1999, this bridge is shaped in the form of hyperboloid and appears as a lightweight glazed tube between two buildings (Collis, 2003). Developed by Smith \& Gill Architects in 2008, Al Masdar Headquarters building in Abu Dhabi is another innovative example formed by eleven hyperboloid-like structures that perform different functions (Figure $1 \mathrm{~b}$ ). These structures not only support the building's curvilinear canopy roof but also act as wind towers creating air movement in the interior courtyard spaces (McKeough, 2008). Likewise, the vault structure of
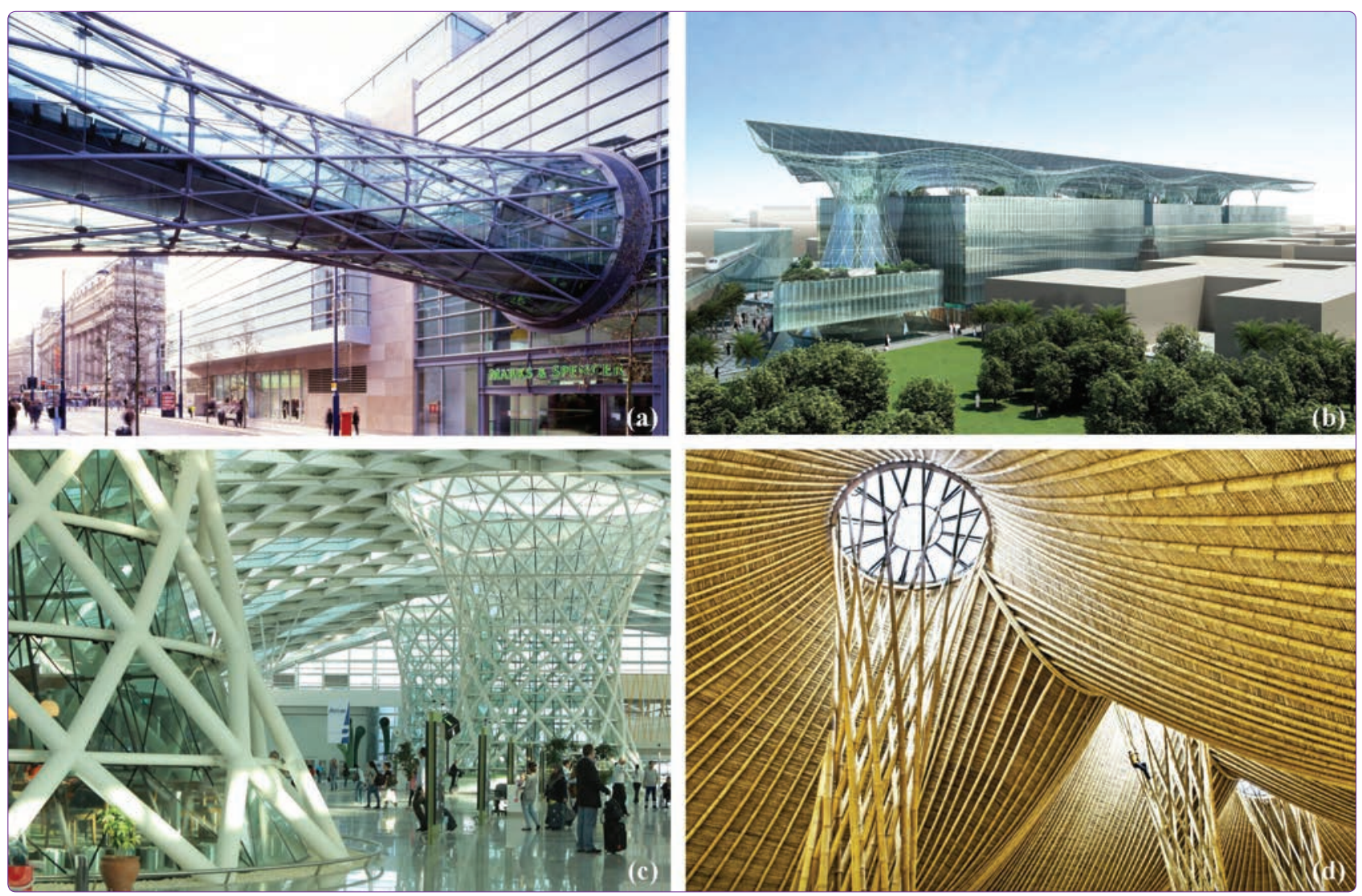

Figure 1. (a) Manchester Corporation Street Footbridge (Photo: Peter Cook); (b) Al Masdar Headquarters Building (Source: Smith \& Gill Architects); (c) Adnan Menderes Airport Domestic Terminal; (d) Three Mountains School (Source: http://kurakurabali.com/islandof-happiness/harmony-with-creation/). 
the Adnan Menderes Airport Domestic Terminal building is supported by four hyperboloid lattice towers that provide natural daylight and ventilation into the interiors (Figure 1c). Similarly, the design of the Three Mountains School building in Bali is dominated by three hyperboloid lattice towers constructed completely from bamboo (Figure 1d). On the other hand, several hyperboloid tower structures serving for different functions have been built around the world such as Barcelona Airport Air Traffic Control Tower, Canton TV \& Sightseeing Tower in China, Tornado Tower in Qatar, Museo Soumaya in Mexico, Kobe Port Tower \& Maritime Museum in Japan and Kisfaludy Observation Tower in Hungary.

The architects of the twenty-first century are challenged to create forms and structures that are more dynamic and adaptive. Rather than relying on conventional structures, they have explored new structural systems that can offer more innovative solutions for architectural applications as changing the building's form. Advances in technology influenced building design techniques and enabled movement to be incorporated into architecture. To produce an architecture that is physically responsive to change, different types of kinetic structures have been developed such as deployable bar structures, folded-plate structures, strutcable structures and membrane structures. Compared to conventional structures, these structures may offer viable solutions for certain types of applications such as exhibition halls, temporary or emergency shelters, retractable roofs for sporting fields and outdoor recreation facilities, protective shelters on excavated archeological sites and responsive facades. Deployable bar structures are impressive examples of the kinetic structures, which not only allow shape transformations like mechanisms in order to adapt to different functions or environmental conditions, but also transfer loads like structures.

Composed of scissor like elements (SLEs), many deployable bar structures having singly curved or synclastic surfaces have been proposed. However, anticlastic hyperboloid and hypar surfaces have been scarcely used for kinetic structures. Earlier works on those surfaces focused on Bennett linkage (Bennett 1903) since it is the only single DOF (degree-of-freedom) 4R (revolute) spatial mechanism that defines a quadric surface with its straight links. However, most of them were restricted to geometric characteristics of the Bennett linkage (Yu 1981; Huang 1997; Baker $2004,2007)$. Some researchers have investigated the construction of deployable structures with the Bennett linkage. Chen and You $(2004,2005)$ have developed structural mechanisms using alternative form of the Bennett linkage. Considering this alternative form, Yu, Luo, and Li (2007) have proposed a deployable membrane structure for aerospace applications. Using equilateral Bennett linkages,
Melin (2005) has proposed a long-span deployable shelter structure housing military helicopters. Moreover, Tian and Chen (2010) have designed a foldable shelter that is composed of five Bennett linkages. Furthermore, Yang, Li, and Chen (2015) have proposed a deployable saddle surface with equilateral Bennett linkages.

Some other researchers have studied possible applications of deployable hyperboloid and hypar structures in architecture. Al Khayar and Lalvani (1998) have explored the application of angulated SLEs to polygonal hyperboloids in order to generate periodic structures (Figure 2a). They have proposed different types of polygonal hyperboloids derived from regular and semi-regular tessellations, but their work was limited to morphological possibilities. Langbecker (2000) has designed a deployable hypar structure composed of translational SLEs, which has a complex system due to the number of links and joints used to generate the desired curvature and deployment. Furthermore, De Temmerman et al. (2007) have developed a deployable hyperboloid mast with angulated SLEs for a temporary membrane canopy structure (Figure $2 \mathrm{~b}$ ). In order to achieve the required structural strength and stiffness, horizontal ties have been added to the mast due to the excessive DOF of the mechanism. Moreover, Escrig and Sanchéz (2013) have proposed a fractal deployable hyperboloid using three segments of scissor units, but the connections of the segments seem problematic due to the eccentricity.

Aforementioned studies have made important contributions to the improvement of deployable hyperboloid and hypar structures. Although many impressive concepts have been proposed varying in size, geometry and retraction methods, they could not been realized in architecture since they are composed of multiple SLEs that require more complex structural and mechanical systems. In those systems, numerous bars have to be connected by single joints in order to generate the desired geometric shapes. This affects the DOF of the whole system and complicate the control of the movement during the deployment process. As the number of bars and joints increases, the structure becomes more complex and the weight and cost of the structure increase. Moreover, new joint types must be developed not only to connect different bars to each other in the structure but also to construct new geometric shapes. Thus, it is crucial to reduce the number of links and joints.

The deficiencies that are inherent in the current designs have led the authors to simplify both geometrical and mechanical complexity of such structures. Using ruled surface generation method, different types of deployable hyperboloids have been constructed and their deployment capacities have been discussed. The proposed solution in this paper leads to a significant reduction of the number of 

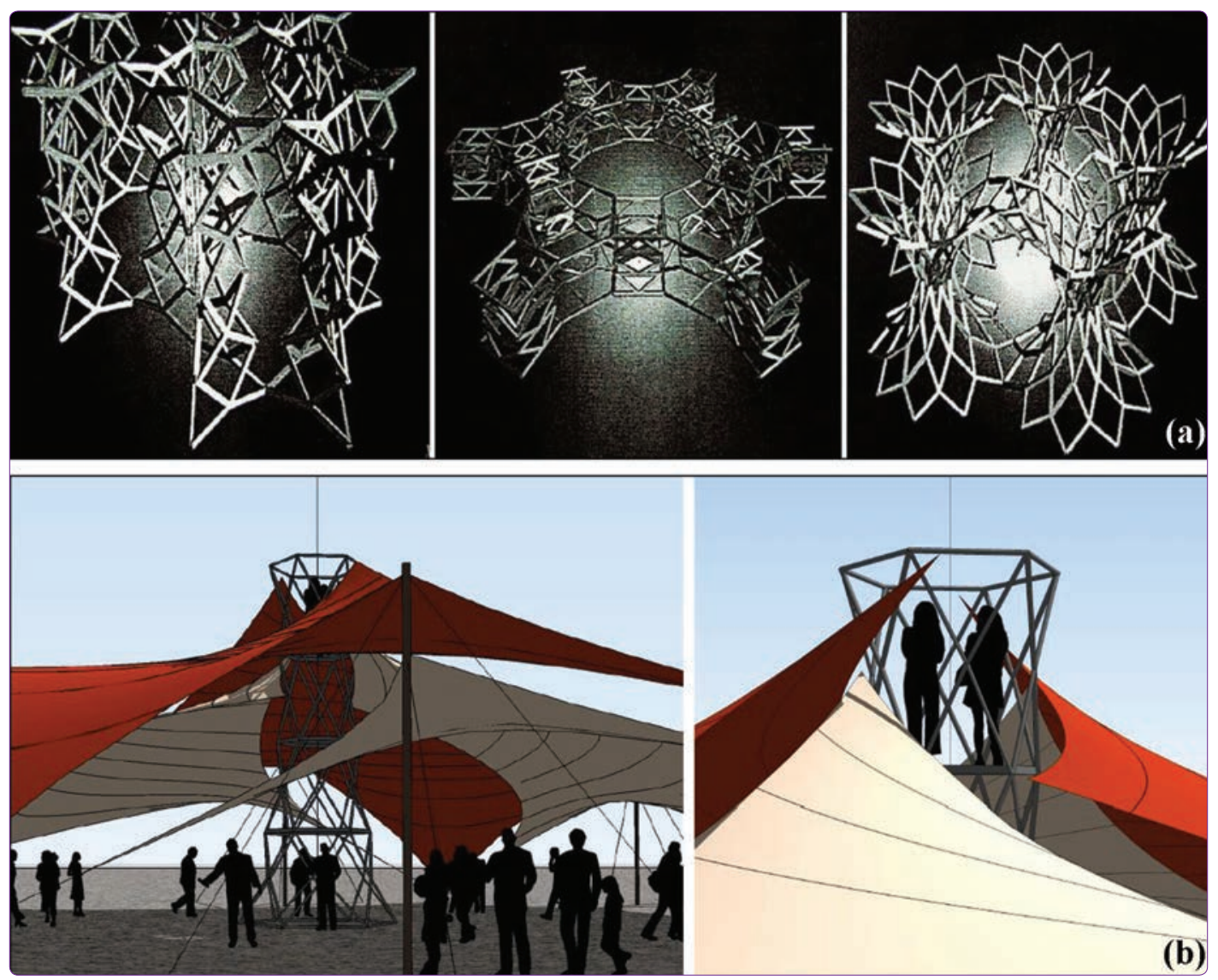

Figure 2. (a) Deployable polygonal hyperboloids (Source: Al Khayar and Lalvani, 1998); (b) Deployable tower (Source: De Temmerman, 2007).

links and joints since the hyperboloid structure can be easily constructed using identical straight bars and joints. By means of the proposed joints, any type of deployable hyperboloid having either identical or different radii of base curves can be built although the structures composed of SLEs require to develop new connection details to generate the geometry.

\section{Geometric Characteristics and Morphology of Hyperboloids}

Hyperboloid of one sheet can be generated by using two different surface generation methods (Pottmann et al. 2007). In the first method, the surface is created by revolving a hyperbola about a central axis. In the second method, a straight line skewed at an angle is revolved about a vertical axis. If the surface has two sets of rulings, the generated surface is called doubly ruled hyperboloid.

To define the doubly ruled hyperboloid geometrically, the following key principles are used. Firstly, two identical base curves (directrix 1 and directrix 2) with a radius of $r$ spaced at a distance $h$ apart are drawn on two horizontal planes as shown in Figure 3a. Secondly, the base curves are divided into $n$ equal parts. It should be noted that the minimum number of $n$ is equal to 3 , because a hyperboloid can be defined with at least three skew lines. Given $n$, the segment angle $(\varphi)$ of the base curves is calculated as

$$
\phi=\frac{360}{n}
$$

Then, the phase angle $\left(\alpha_{k}\right)$ of the skew lines on $x y$-plane is defined. $\alpha_{k}$ must be less than $180^{\circ}$, otherwise the generated surface becomes a cone. Therefore, the choice of $\alpha_{k}$ is limited. Possible phase angles can be found by the formula

$$
\alpha_{k}=k \cdot \frac{360}{n}
$$

where $k=1,2, \ldots,[n / 2]$ for $n$ odd, $k=1,2 \ldots,[n / 2]-1$ for $n$ even and $[()]$ is the floor function.

According to the chosen phase angle, a skew line $(I)$ is drawn from the $i^{\text {th }}$ node on the bottom base curve up to the $(i+k)^{t h}$ node on the top base curve. Then, the skew line is revolved clockwise around the z-axis with the chosen phase angle as depicted in Figure $3 \mathrm{~b}$. Thereafter, the same skew line that is mirrored about the vertical axis is revolved counterclockwise with the same twist angle ( $\beta$ ) (Figure $3 c$ ). 


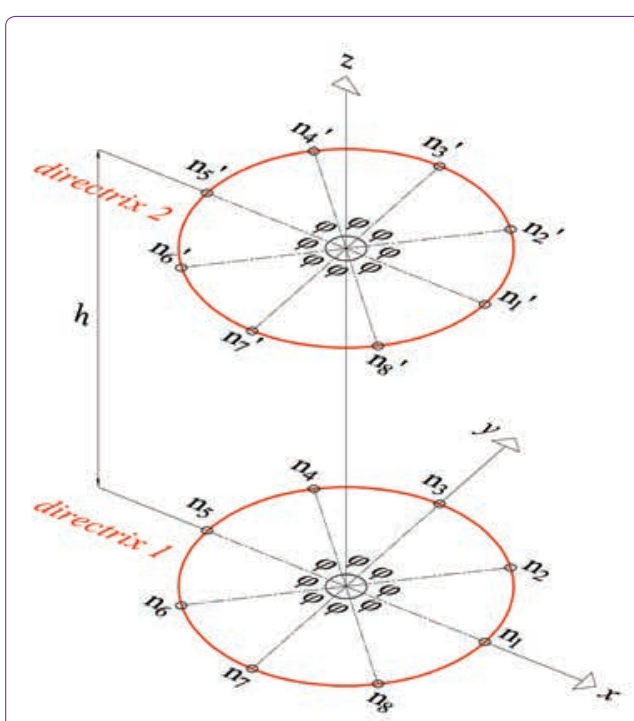

(a)

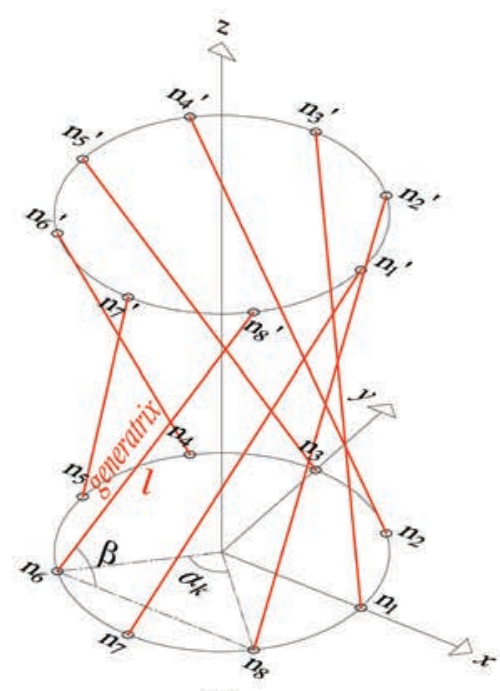

(b)

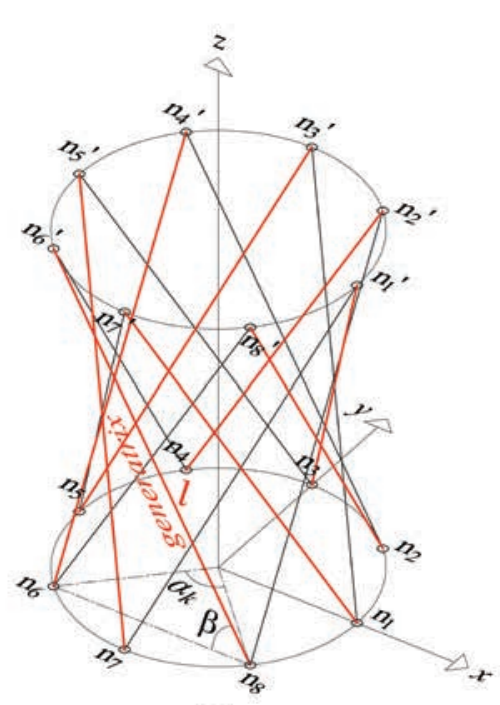

(c)

Figure 3. (a-c) Generation process of doubly ruled hyperboloid.

According to the parameters $h, r$ and $n$, the $x y z$-coordinates of the nodes on the bottom and top base curves are found. These are named as $X_{n i}, Y_{n i}{ }_{n i}$ and $X_{n i}{ }^{\prime}, Y_{n i}{ }^{\prime}, Z_{n i}{ }^{\prime}$ respectively. Because the bottom and top base curves are identical, $x$ - and $y$-coordinates of the aforementioned nodes are the same, but $z$-coordinates are different. According to Figure $4 a, x y z$-coordinates of the $i^{t \text { th }}$ node (where $i=1,2,3, \ldots, n)$ are as follows:

$$
\begin{aligned}
& X n_{i}=X n_{i}{ }^{\prime}=r \cdot \cos [(i-1) \cdot \varphi] \\
& Y n_{i}=Y n_{i}{ }^{\prime}=r \cdot \sin [(i-1) \cdot \varphi] \\
& Z n_{i}=0 \\
& Z n_{i}{ }^{\prime}=h
\end{aligned}
$$

Revolving the skew lines in clockwise and counterclockwise directions exposes intersections between those lines and the base curves as shown in Figure $4 \mathrm{~b}$. The number of intersections depends on the parameters $n$ and $\alpha_{k}$. The number of skew lines $\left(I_{n}\right)$, intersections on each skew line $\left(J_{1}\right)$, intermediate intersections on each skew line $\left(J_{i}\right)$ and the total number of intersections on hyperboloid surface $\left(J_{h}\right)$ can be found as:

$$
\begin{aligned}
& l_{n}=2 n \\
& J_{l}=2 k+1 \\
& J_{i}=J_{l}-2 \\
& J_{h}=n \cdot J_{l}
\end{aligned}
$$

where $n$ is the number of nodes on the base curve and $k$ is the factor of $\alpha_{k}$. For instance, in an octagonal hyperboloid with a phase angle of $\alpha_{k}=\alpha_{3}=135^{\circ}$, the parameter $k$ is equal to 3 . Therefore, $J_{l}=2 \times 3+1=7, J_{i}=7-2=5$ and $J_{h}=$ $8 \times 7=56$.
To calculate the coordinates of the nodes at intermediate intersections, an equation for the skew lines needs to be defined. Since the coordinates of the nodes on the bottom and top base curves are given by Eq. (3), a general formula can be written for two intersecting skew lines.

$$
\frac{x_{J_{i}}-x_{n_{i}}}{x_{k_{i}}-x_{n_{i}}}=\frac{y_{J_{i}}-y_{n_{i}}}{y_{k_{i}}-y_{n_{i}}}=\frac{z_{J_{i}}-z_{n_{i}}}{z_{k_{i}}-z_{n_{i}}}=t
$$

Due to the curvature of the hyperboloid, the distances between intersection points $\left(d_{i}\right)$ are not identical and can be calculated by the formula

$$
d_{i}=\sqrt{\left(x_{J_{i+1}}-x_{J_{i}}\right)^{2}+\left(y_{J_{i+1}}-y_{J_{i}}\right)^{2}+\left(z_{J_{i+1}}-z_{J_{i}}\right)^{2}}
$$

In order to study the morphology of the hyperboloid, a parametric model is constructed in Grasshopper ${ }^{\circledR}$. The geometrical conditions of the hyperboloid surface are defined with a set of variables in the parametric model. The model allows generating different types of hyperboloids by changing the input parameters $h, r, n$ and $\alpha_{k}$. For instance, $h$ or $r$ can be adjusted to define the size of the hyperboloid. Figure 5 illustrates the process of changing the parameter $r$ in which the base curves have same radius.

In addition to the hyperboloids generated with identical base curves, it is possible to obtain hyperboloids with different radii of base curves. As shown in Figure 6, the radius of the bottom base curve $\left(r_{b}\right)$ or the radius of the top base curve $\left(r_{t}\right)$ can be changed. In Figure $6 a, r_{t}$ is kept constant and $r_{b}$ is changed. On the contrary, in Figure $6 b, r_{b}$ is kept constant and $r_{t}$ is changed.

Similarly, the curvature can be changed by increasing or decreasing the parameter $\alpha_{k}$. If $\alpha_{k}$ is increased, the number of intersections between the skew lines increases as well 


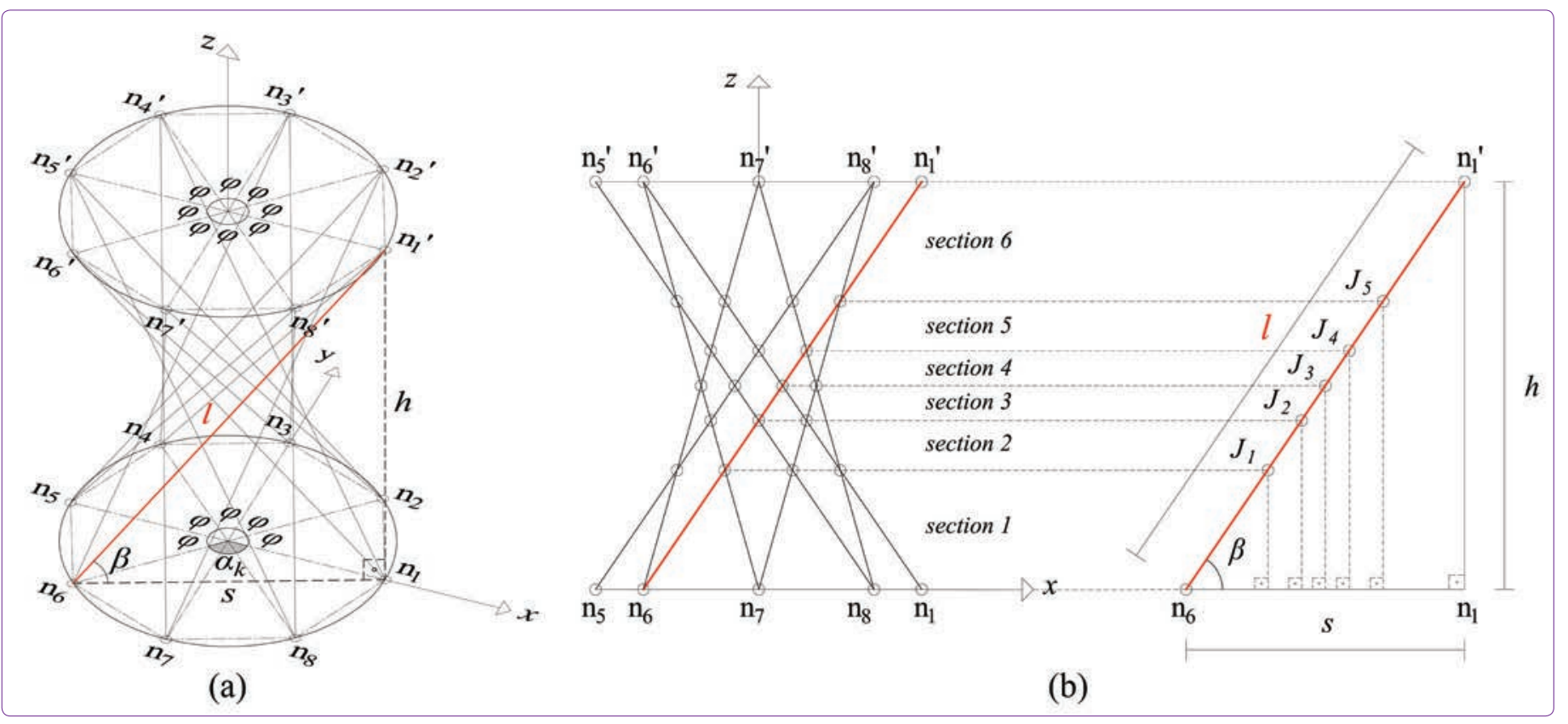

Figure 4. (a) Construction parameters of hyperboloid; (b) intersection points of skew lines.

(Figure 7a-e). However, when $\alpha_{k}=180^{\circ}$, all the lines intersect at a single mid-point and a conical surface is obtained as shown in Figure 7f. By means of the aforementioned surface generation method, different types of hyperboloids are generated. In Figure 8 , some basic types are given with their construction parameters.

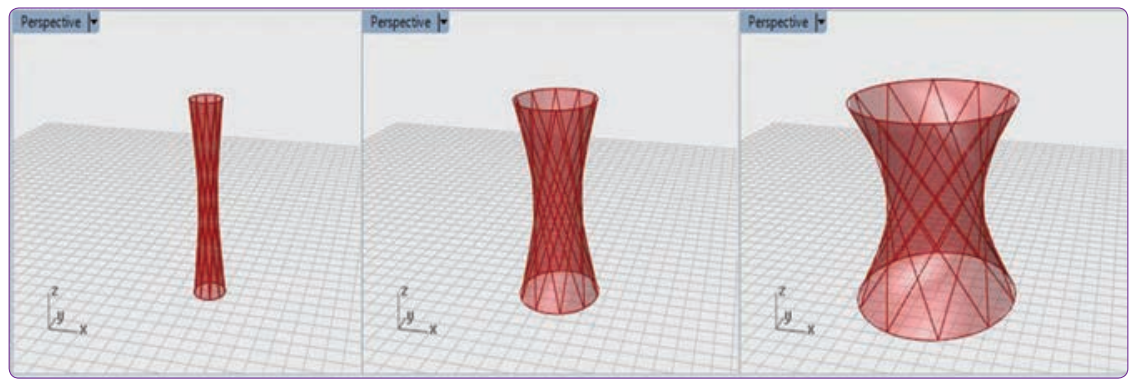

Figure 5. Decagonal hyperboloid generated by changing $r$.

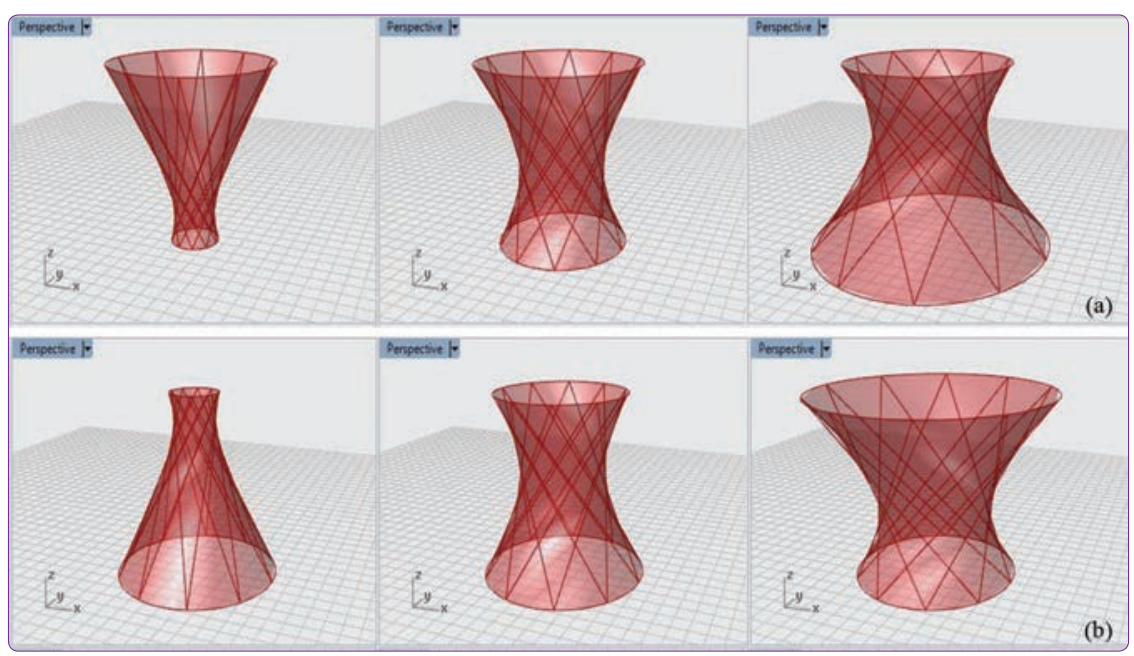

Figure 6. Decagonal hyperboloid: (a) generated by changing $r_{b^{\prime}}$ (b) generated by changing $r_{t^{*}}$ 


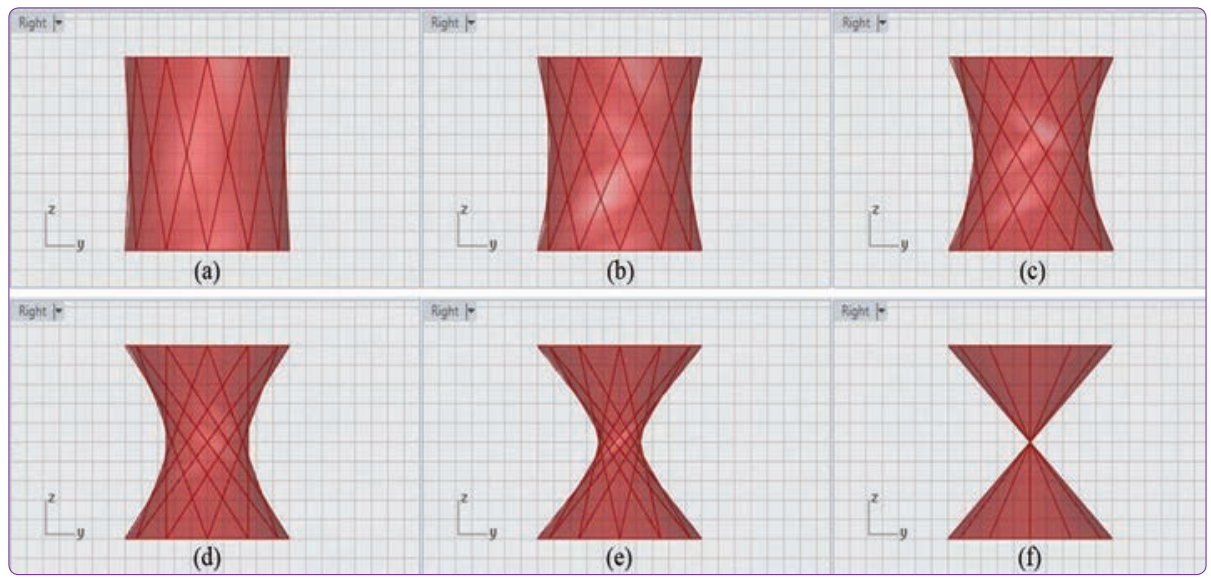

Figure 7. Dodecagonal hyperboloid generated by changing ak: (a) $J_{i}=1 ;$ (b) $J_{i}=3 ;$ (c) $J_{i}=5$; (d) $J_{i}=7$; (e) $J_{i}=9$; (f) $J_{i}=1$.

\section{Construction of Deployable Doubly Ruled Hyperboloids}

Hyperboloid is a quadric surface. Generating the hyperboloid by ruled surface generation method also introduces sub-quadric surfaces between the intersection points. Therefore, at first, the possibility of constructing deploy- able hyperboloids with the Bennett linkage has been investigated. Obeying the geometric design principles of the linkage and connecting the Bennett loops to each other, various structural mechanisms (SMs) have been constructed (Figure 9). It has been realized that the SMs can deploy into cylindrical shapes when the end links stand free. How-

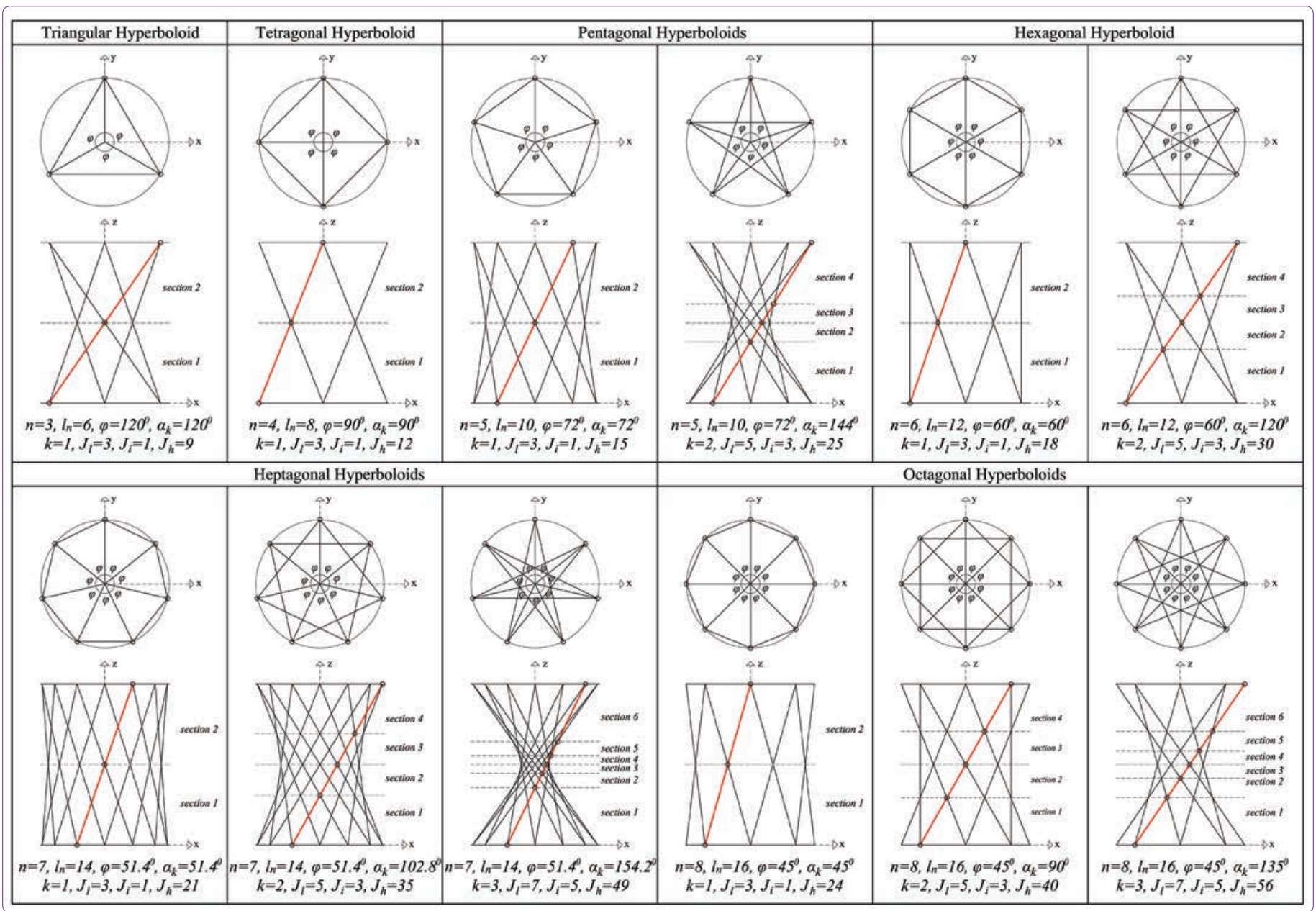

Figure 8. Types of doubly ruled hyperboloids. 


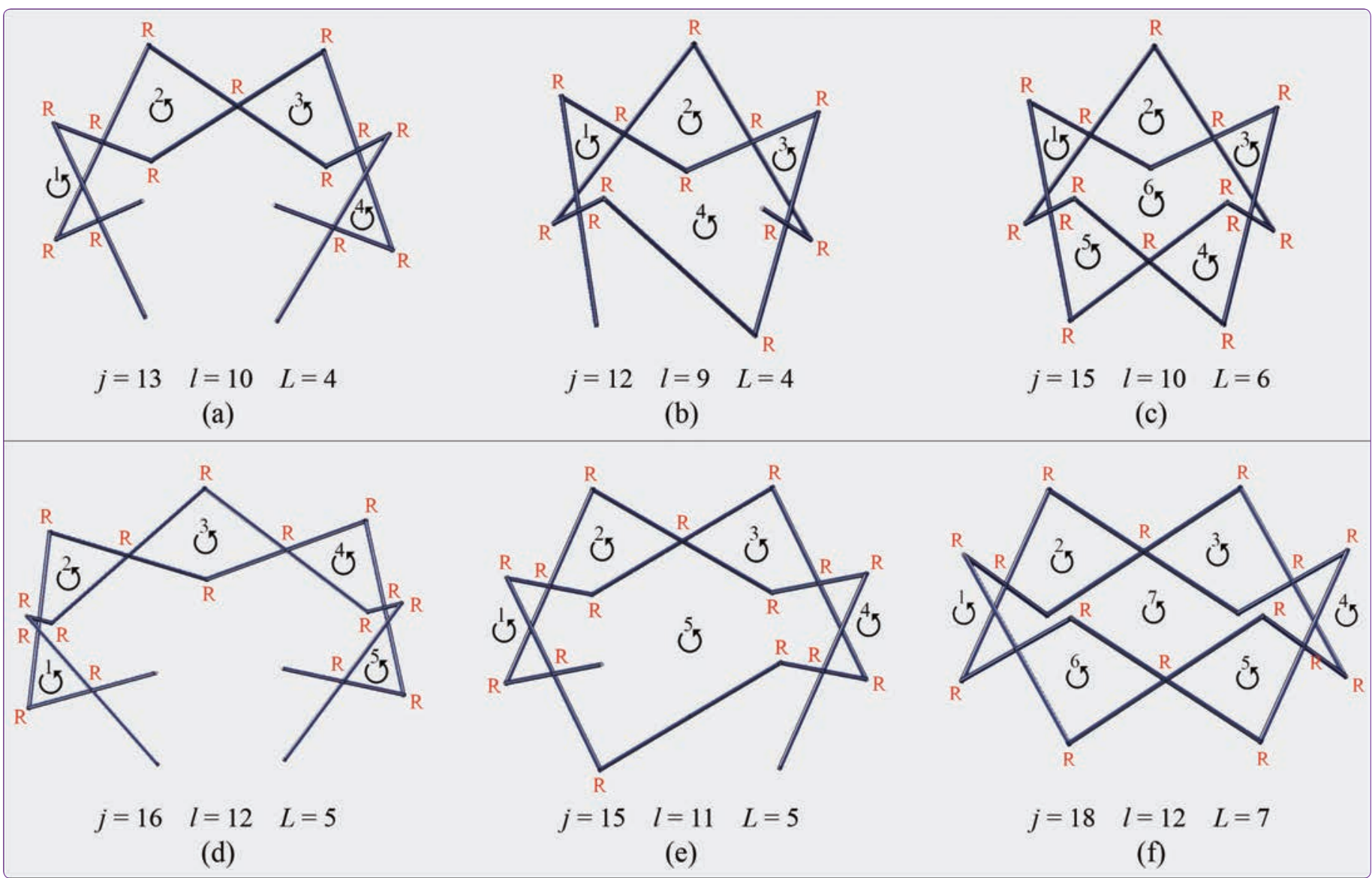

Figure 9. SMs composed of Bennett linkages.

ever, they become static structures and do not allow any motion when the end links are connected to each other. This argument has been proved by calculating the mobility of the SMs according to the formula proposed by Alizade (2010), which is used for multi-loop mechanisms.

$$
M=\sum_{i=1}^{j} \mathrm{f}_{\mathrm{i}}-\sum_{k=1}^{L} \lambda_{k}+q-j_{p}
$$

where $j$ is the number of joints, $f_{i}$ is the relative joint motion, $L$ is the number of independent loops, $\lambda_{k}$ is the number of independent loop closure equations, $q$ is the number of excessive elements (links or joints) and $j_{p}$ is the number of passive joints.

The Bennett linkage operates in $\lambda=3$; therefore $\lambda$ is taken as 3 for each Bennett loop when calculating the mobility of the SMs. For the SMs composed of four and five Bennett loops as indicated in Figures $9 a$ and $9 d, M=13-(3 \times 4)=$ 1 and $M=16-(3 \times 5)=1$ respectively. If one of the end links is eliminated and then the other end link is connected to the system as depicted in Figures $9 \mathrm{~b}$ and $9 \mathrm{e}$, the mobility of the systems becomes $M=12-(3 \times 4)=0$ and $M=15-(3 \times 5)$ $=0$, respectively. If the eliminated link is added again to the system as shown in Figures $9 \mathrm{c}$ and $9 \mathrm{f}$, one finds that $M$ $=15-(3 \times 6)=-3$ and $M=18-(3 \times 7)=-3$. Similar approach has been applied to the SMs that consist of $n$ number of Bennett loops. Mobility has been again found as -3 for those SMs. In addition to the mobility calculations, motion analyses of those SMs have been performed in SolidWorks ${ }^{\circledR}$ and CATIA $^{\circledR}$ to check mobility. The results show that the SMs become a preloaded structure when all the loops are joined together in order to form a hyperboloid with $J=1$. Thus, it can be said that it is not possible to construct a deployable hyperboloid with the Bennett linkage. Because the system requires the use of different types of joints at the nodes, it is necessary to develop a new design solution.

Before introducing the solution, whether or not the distances between the intersection points change during the deployment process needs to be investigated. For this purpose, many models have been constructed and analyzed. Using Eqs. (3), (8) and (9), those distances have been calculated at different configurations of the hyperboloids. It has been found that distances do not change during the deployment process. In order to prove this argument, one of the constructed models was selected. An octagonal hyperboloid with $J_{i}=3$ is analyzed at three different configurations (Figure 10). With respect to the given parameter $I=$ $5.745 \mathrm{~m}$, the parameters $r$ and $h$ in configurations 1,2 and 

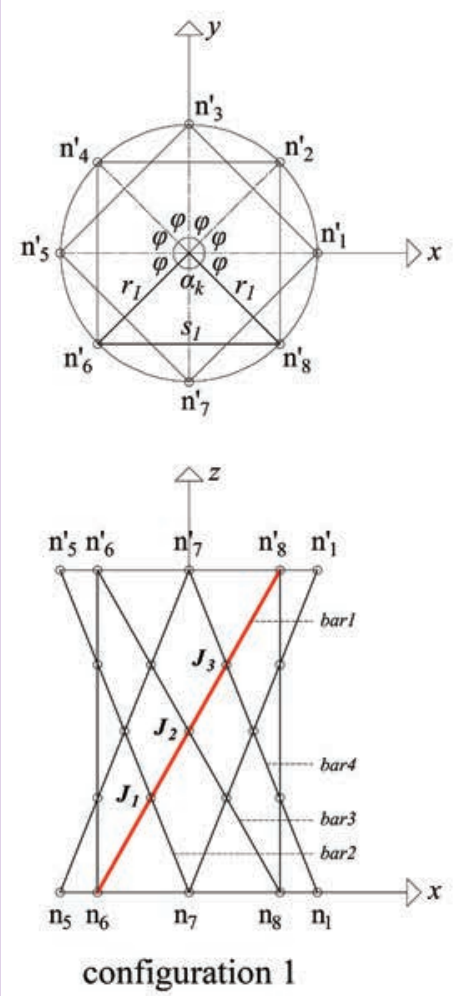
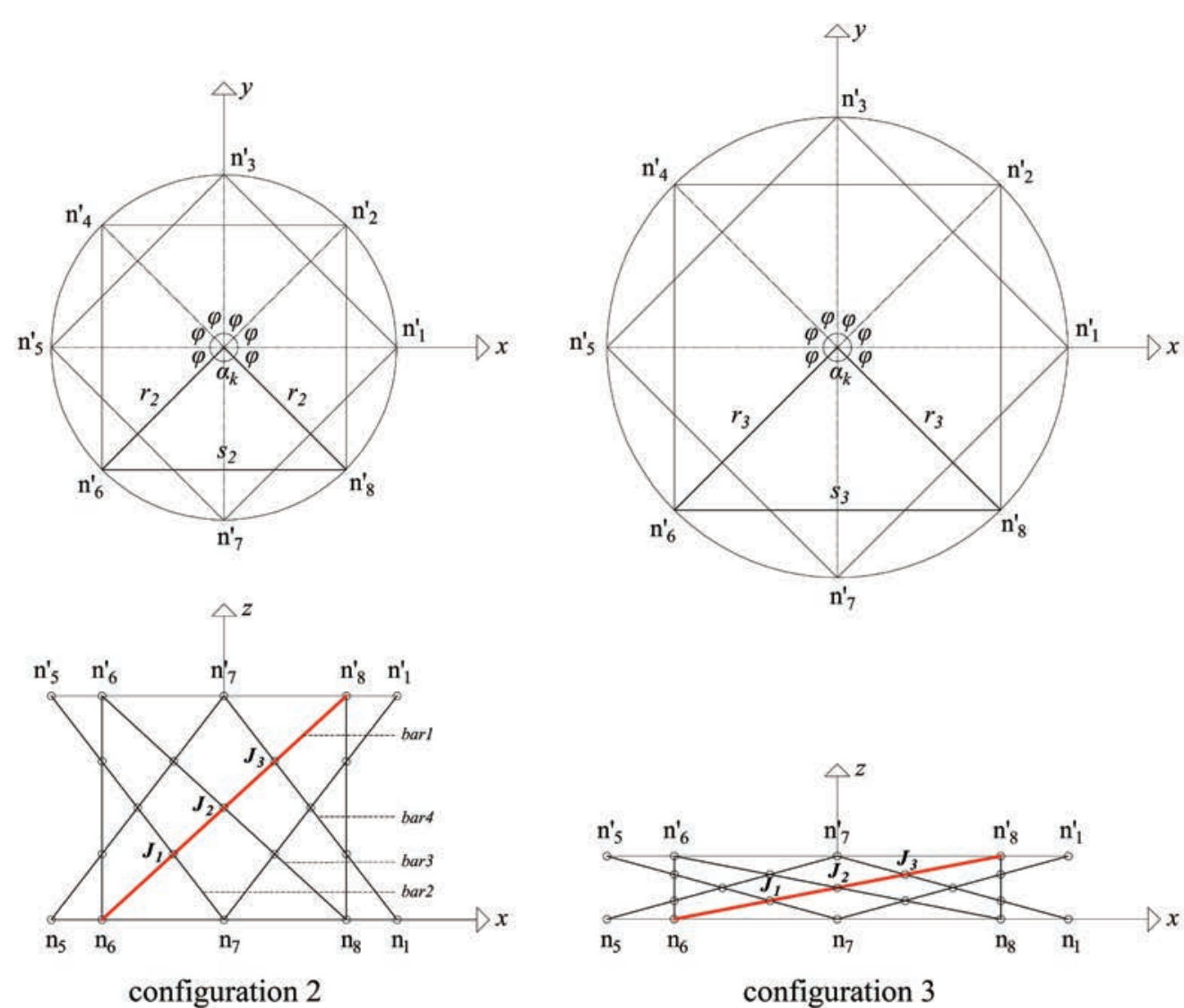

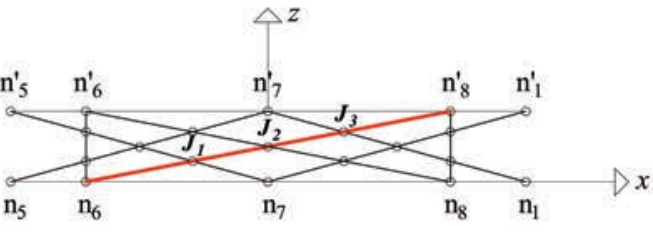

configuration 3

Figure 10. Three different deployed configurations of octagonal hyperboloid.

3 are respectively as follows: $r_{1}=2 \mathrm{~m}, h_{1}=5 \mathrm{~m}, r_{2}=3 \mathrm{~m}, h_{2}$ $=3.87 \mathrm{~m}, r_{3}=4 \mathrm{~m}$ and $h_{3}=1 \mathrm{~m}$. The $x y z$-coordinates of the top, intermediate and bottom nodes on bar1, bar2, bar3 and bar4 in each configuration have been calculated according to Eqs. (3) and (8). The values are given in Table 1. According to Eq. (9), the distances between the selected nodes $\left(n_{6}-J_{1}, J_{1}-J_{2}, J_{2}-J_{3}\right.$ and $\left.J_{3}-n_{8}{ }^{\prime}\right)$ have been calculated. It has been found that $d_{n 6 / 1}=1.68, d_{J 1 / 2}=1.18, d_{J 2 / 3}=1.18$ and $d_{J 3 n 8}{ }^{\prime}=1.68$ for configurations 1,2 and 3 . The result shows that those distances do not change during the deployment process. Therefore, it can be claimed that deployable hyperboloids can be easily constructed by the joints that have only rotational motion.

Considering that the lengths of the bars between the intersection points remain constant during the deployment process, a SM composed of only $R$ and spherical (S) joints has been developed. The axes of bars intersect; therefore, the center of rotation is the same for two intersecting bars. $R$ joints have been used at mid-intersections and $S$ joints have been used at top, bottom, and intermediate intersections of the concurrent bars as illustrated in Figure 11a. The $R$ joints restrict the movements of the bars. Their axes lie in the same plane and intersect at a single point (Figure $11 b)$. Because the $S$ joint has 3-DOF due to three rotational movements about the intersecting rotation axes, it allows the hyperboloid to deploy from one configuration to another.

Thereafter, the mobility of the hyperboloids has been investigated. For this purpose, at first, the loops on the hyperboloid surface have been analyzed. As shown in Figure 11c, the hyperboloid has top, bottom and surface loops. The number of loops depends on the type of hyperboloid. Because the bottom loop has been considered as a dependent loop, only the top and surface loops have been taken into account in the mobility formula. However, the top and surface loops have different $\lambda$. While the top loop belongs to $\lambda_{t}=6$ system which describes three rotational and three translational active motion, the surface loops belong to either $\lambda_{s}=5$ system allowing three rotational and two translational active motion or $\lambda_{s}=4$ system allowing three rotational and one translational active motion in subspace. If the bars have only one intersection at the middle $\left(J_{i}=\right.$ 1), $\lambda$ for the surface loops is $\lambda_{s}=5$. But, if $J_{i}>1$, it is equal to $\lambda_{s}=4$. In that condition, the system has some excessive joints. To calculate the excessive joints of the hyperboloid (q), a new formula has been introduced.

$$
q=n \cdot\left(J_{i}-\lambda_{s}\right)+\lambda_{t}+1
$$




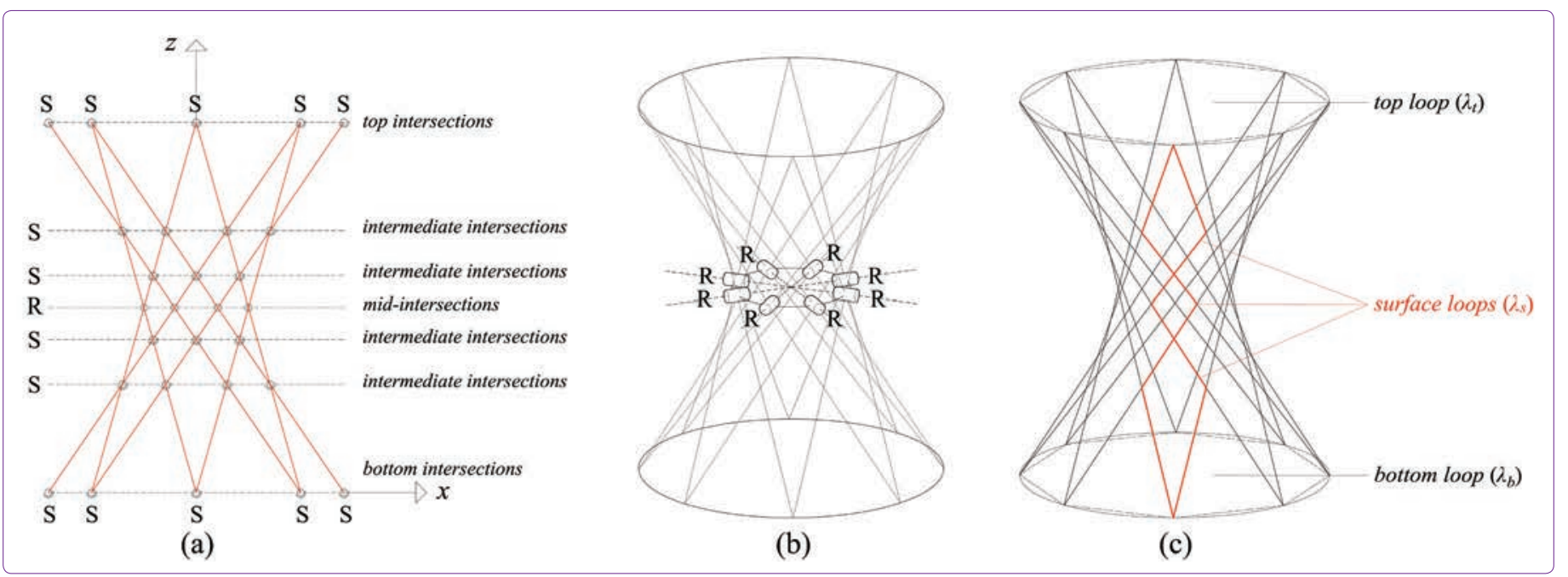

Figure 11. (a) Joint types at intersection points; (b) axes of R joints; (c) loops on hyperboloid surface.

where $n$ is the number of nodes on the base curve, $J_{i}$ is the number of intermediate intersections on the link, $\lambda_{s}$ is the DOF of the independent surface loops and $\lambda_{t}$ is the DOF of the independent top loop. For instance, for the hyperboloids in which each bar has 5 intersections, $J_{i}=5-2$ $=3$ according to Eq. (6). Due to $J_{i}>1, \lambda_{s}=4$ for the surface loops and $\lambda_{t}=6$ for the top loop. The number of excessive joint is written as $q=n \cdot(3-4)+6+1=7-n$.

After determining the parameter $q$, different types of hyperboloids have been constructed and analyzed in terms of their mobility. The system parameters of the hyperboloids are given in Table 2. It has been found that the triangular hyperboloid deploys with single DOF while the other hyperboloids with $J_{i}=1$ deploy with multi DOF (M-DOF). This argument was proved by Eq. (10). According to Table 2 , the mobility of the triangular hyperboloid has been calculated as $M=21-[(5 \times 3)+(5 \times 1)]+0-0=1$. The system can deploy from a closed configuration to an expanded one as shown in Figure 12a. In tetragonal hyperboloid, it has been found that $M=28-[(5 \times 4)+(6 \times 1)]+0-0=2$. Because the tetragonal hyperboloid has 2-DOF, it can deploy both $x$ - and $y$-directions independently. Similarly, in pentagonal hyperboloid, the mobility of the system is equal to $M=$ $35-[(5 \times 5)+(6 \times 1)]+0-0=4$. Likewise, the mobility of the hexagonal hyperboloid is $M=42-[(5 \times 6)+(6 \times 1)]+0-0=6$.

Based on aforementioned calculations, it can be claimed that the mobility of the one-mid-intersection hyperboloids increases as the number of nodes on the base curve increases. However, it has been realized that the mobility of the M-DOF hyperboloids that have more than one intermediate intersection decrease to 1 again due to the fact that $L$ increases as $J$ increases. To analyze the mobility of the hyperboloids with $J_{i}>1$, different types of hyperboloids have been constructed and mobility calculated according to Eq. (10).
In pentagonal hyperboloid with $J_{i}=3$, the mobility has been calculated as $M=65-[(4 \times 15)+(6 \times 1)]+2-0=1$. In hexagonal hyperboloid with $J_{i}=3$, the mobility of the system equals to $M=78-[(4 \times 18)+(6 \times 1)]+1-0=1$. In Figure $12 b$, the deployment process of the single DOF hexagonal hyperboloid is shown. Similarly, in heptagonal hyperboloid with $J_{i}=3$, it has been found that $M=91-[(4 \times 21)+(6 \times 1)]+0-0$ $=1$. In heptagonal hyperboloid with $J_{i}=5, M=$ $133-[(4 \times 35)+(6 \times 1)]+14-0=1$. By using the same method of calculation, the mobility of different types of doubly ruled hyperboloids can be found. Consequently, it can be claimed that the only single DOF hyperboloid among the examples of hyperboloids with $J_{i}=1$ is the triangular hyperboloid. The others belong to M-DOF system. However, all the hyperboloids with $J_{i}>1$ belong to single DOF system in which they can deploy from a compact configuration to an extended one.

\section{Conclusion and Discussion}

Since the hyperboloid structures can be easily constructed from straight fragments and resist to extreme forces owing to their curved form, they have been employed in many buildings having different functions. When the kinetic structures came into vogue in the last decades, it has attracted the interest of both architects and engineers due to their ability to change their geometric from one configuration to another one and their potentials for compact storage and transportability. Various alternative solutions have been proposed for their architectural applications. However, rather than constructing the hyperboloid geometry by straight fragments, SLEs have been used which increased the complexity, weight and cost of the structures. In those solutions, numerous bars have interconnected by single joints and different types of connection details developed to solve the geometric incompatibilities between 


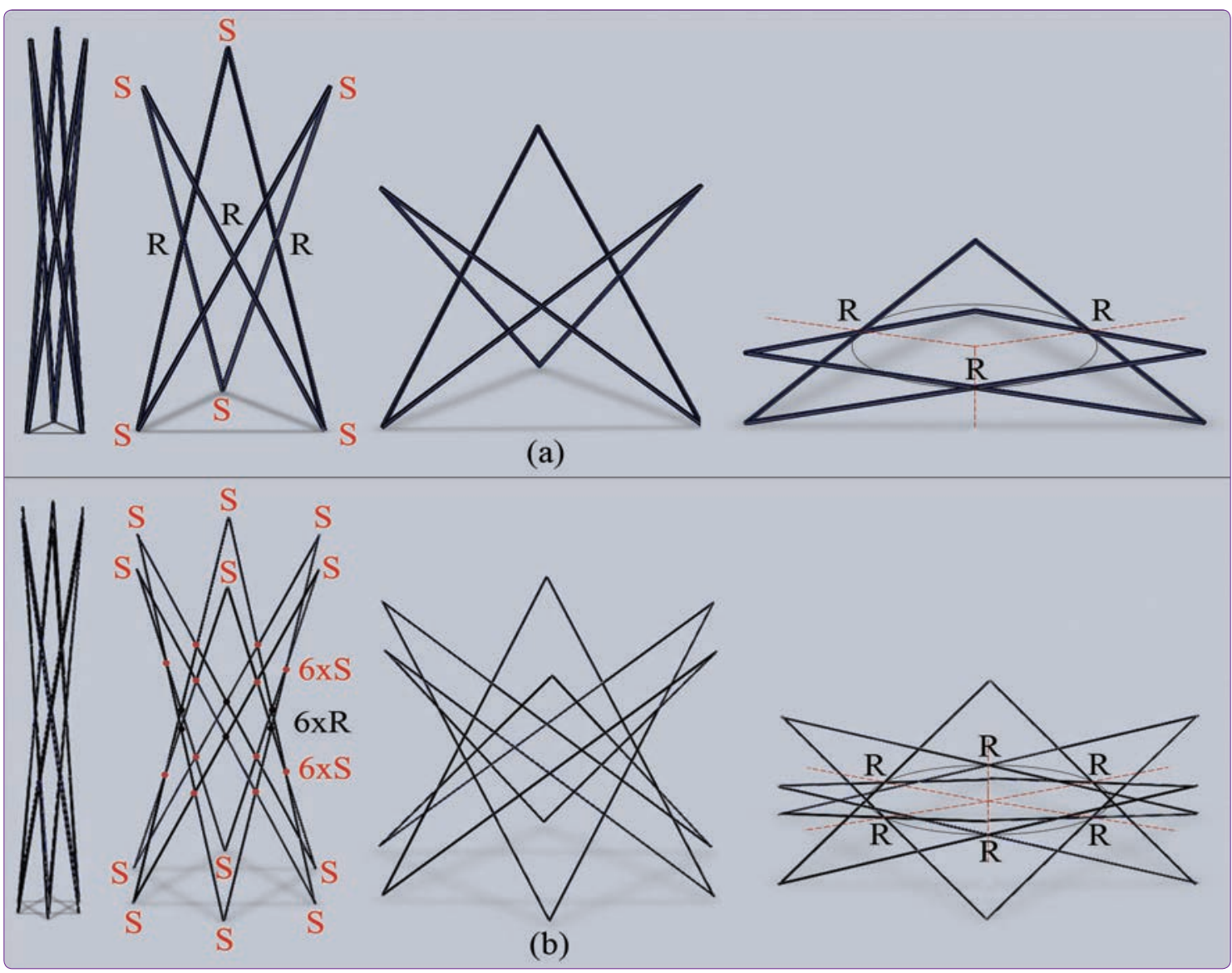

Figure 12. (a) Deployment process of single DOF hyperboloids: (a) triangular hyperboloid; (b) hexagonal hyperboloid with $J_{i}=3$.

the members that occur during the deployment process. Due to the mechanical complexity of their systems and lack of structural resistance against design loads, the proposed structures could not been realized in architecture.

In this paper, a method has been presented in order to build deployable hyperboloids. Starting from the analysis of the geometric principles of doubly-ruled hyperboloids, the morphology has been discussed in detail. It has been demonstrated the relation between the curvature of the surface and the rotation angle of the skew lines. By means of the parametric model built in Grasshopper ${ }^{\circledR}$, many doubly-ruled hyperboloids having different curvatures have been generated. Considering the geometric requirements of the hyperboloid surface, it has been attempted to built the deployable hyperboloids by using the current mechanisms in literature. For this purpose, hyperboloids have been constructed with respect to the geometric design principles and special conditions of Bennett linkage since it defines a quadric surface with its straight links. However, it has been proved according to the kinematic studies that the constructed hyperboloids with the Bennett linkages are not deployable. Thus, a new SM has been proposed to obtain deployable doubly ruled hyperboloids.
It has been demonstrated that the deployment behavior of SM is directly related to joint types that are used at the intersection points of the bars. According to the proposed joint types, kinematic studies of the hyperboloids have been carried out. Based on kinematic studies, the mobility and deployment capabilities of the constructed models have been discussed. It has been found that the triangular hyperboloid and the hyperboloids with $J_{i}>1$ have single DOF while the other hyperboloids with $J_{i}=1$ have M-DOF. Finally, it has been proved that deployable hyperboloids with single DOF, whose number of intermediate intersection is more than one, can be constructed with only $R$ and $S$ joints. By this means, it has been achieved to obtain hyperboloids by identical straight bars and joints. The proposed hyperboloids offer an advantage over existing solutions of the deployable bar structures composed of SLEs, because it reduces the number of elements and the joints to be used in the system to create the structure. Thereof, the complexity, weight and the cost of the structure can be reduced.

The proposed deployable hyperboloids can be used in various applications where form flexibility is required to adapt to changing circumstances. For instance, they can be used 
as a self-standing observation tower structure on which several platforms are attached. The height of the structure can be changed according to the view. Moreover, they can serve as deployable masts for a temporary canopy structure. As the hyperboloids deploy, the geometric shape of the canopy can be changed according to the user demands. Apart from using hyperboloid as a self-standing structure, a number of hyperboloid sections can be joined together to create new geometric forms that serve as a shelter structure. As the deployable hyperboloids provide desired flexibility and strength, other alternative solutions can be proposed for both permanent and temporary structures.

\section{References}

Al Khayer, M., and Lalvani, H. (1998) "Scissor-Action Deployables Based on Space-Filling of Polygonal Hyperboloids," IUTAMIASS Symposium on Deployable Structures: Theory and Applications, Kluwer Academic Publishers, Cambridge, UK, pp. 1-10.

Alizade, R. (2010) "Structural Synthesis of Robot Manipulators," Proc. AZcIFToMM 2010 International Symposium of Mechanism and Machine Science, Izmir, pp. 11-32.

Baker, J. E. (2004) “The Composition of Bennett's Hyperboloids from the Loop Itself," Journal of Mechanical Design, 126(5), pp. 875-880. DOI: 10.1115/1.1767183

Baker, J. E. (2007) "Kinematic Investigation of the Deployable Bennett Loop." Journal of Mechanical Design, 129(6), pp. 602-610. DOI: 10.1115/1.2717229

Bennett, G. T. (1903) “A New Mechanism.” Engineering 76, pp. 777-778.

Chen, Y., and You, Z. (2004) “Deployable Structure," U.S. Patent Application No. 0120758 A1.

Chen, Y., and You, Z. (2005) "Mobile Assemblies based on the
Bennett Linkage," Proc. R. Soc. A, 461, pp. 1229-1245. DOI: 10.1098/rspa.2004.1383

Collis, H. (2003) Transport, Engineering and Architecture. Oxford: Architectural Press.

De Temmerman, N. (2007) "Design and Analysis of Deployable Bar Structures for Mobile Architectural Applications," Ph.D. Thesis, Vrije Universiteit Brussel.

Escrig, F., and Sanchéz, J. (2013) "Fractal Deployable Umbrella," Proc. of the First Conference Transformables, Editorial Starbooks, Seville, Spain, p. 231.

Huang, C. (1997) "The Cylindroid Associated with Finite Motions of the Bennett Mechanism." Journal of Mechanical Design, 119(4), pp. 521-524. DOI: 10.1115/1.2826399

Langbecker, T. 2000. "Kinematic and Non-linear Analysis of Foldable Scissor Structures." PhD diss., University of Queensland, Brisbane, Australia.

McKeough, T. 2008. "AS+GG Tapped for Ultra-Green Project in UAE." Architectural Record, May 7.

Melin, N. (2005) "Application of Bennett Mechanisms to LongSpan Shelters," PhD diss., University of Oxford, UK.

Pottmann, H., Asperl, A., Hofer, M. and Kilian, A. (2007) Architectural Geometry. Exton, Pa.: Bentley Institute Press.

Tian, P., and Chen, Y. (2010) "Design of a Foldable Shelter," Proc. of the International Symposium of Mechanism and Machine Theory - AzCIFToMM, Izmir, pp. 102-106.

Yang, F., Li, J. and Chen, Y.(2015) "A Deployable Bennett Network in Saddle Surface," The 14th IFToMM World Congress, Taipei, Taiwan. DOI: 10.6567/IFToMM.14TH.WC.OS8.015

Yu, H-C. (1981) "The Bennett Linkage, Its Associated Tetrahedron and the Hyperboloid of Its Axes," Mechanism and Machine Theory 16(2): 105-114. DOI: 10.1016/0094-114X(81)90056-2

Yu, Y., Luo, Y. and Li, L. (2007) "Deployable Membrane Structure Based on the Bennett Linkage," Proc. IMechE, Part G: J. Aerospace Eng. 221(5), pp. 775-783. DOI: 10.1243/0954410 